\title{
A visão da enfermagem sobre o atendimento humanizado no setor de urgência e emergência
}

\author{
Nursing's view on humanized care in the urgent \\ and emergency sector
}

\author{
Sabrina Vasques Cézar ${ }^{1}$, Janaína Samantha Martins de Souza²
}

\section{Resumo}

Objetivo: analisar a visão da enfermagem sobre o atendimento humanizado no setor de urgência e emergência e identificar fatores que dificultem esse processo. Material e Método: trata-se de um estudo descritivo, com abordagem quantitativa, realizado em uma instituição hospitalar privada localizada no Sul do Brasil. Os dados foram recolhidos através de questionários formulados por 20 perguntas, nos quais foram coletadas informações referentes à identificação do profissional como: gênero, faixa etária, escolaridade, materiais relativos ao conhecimento e visão da equipe em relação ao atendimento humanizado. Posteriormente, os materiais foram analisados e organizados em tabelas, os quais foram computados no programa SPSS ${ }^{\circledR}$ - Statistical Package for the Social Sciences (SPSS ${ }^{\circledR} 20$ ). Resultados: considerando os critérios de inclusão e exclusão, a amostra foi composta por 16 questionários válidos. Os dados analisados evidenciaram que a equipe tem compreensão sobre o atendimento humanizado e desejo em adquirir mais conhecimento, porém há insatisfação devido à sobrecarga de trabalho ocasionada com a superlotação do serviço. Conclusão: a pesquisa comprovou que os profissionais enfrentam dificuldades durante sua jornada de trabalho devido à alta demanda de atendimentos, evidenciando-se, assim, a necessidade da administração de estratégias para situações de fluxo intenso e a carência de profissionais para o setor de urgência e emergência. Palavras-chave: Acolhimento. Ética em enfermagem. Pacientes. Profissionais de enfermagem.

\begin{abstract}
Objective: to analyze the nursing vision on humanized attendance in the urgent and emergency sector and identify factors that make this process difficult. Material and Method: this is a descriptive study, with a quantitative approach, carried out in a private hospital institution located in the South of Brazil. The data were collected through a twenty-question questionnaire, inquiring information referring to the professional's identification such as gender, age group, schooling, materials related to the team's knowledge and vision concerning the humanized attendance. Later, the materials were analyzed and organized in tables, computed in the program SPSS ${ }^{\circledR}$ - Statistical Package for the Social Sciences (SPSS $\left.{ }^{\circledR} 20\right)$. Results: considering the inclusion and exclusion criteria, the sample consisted of 16 valid questionnaires. The data analyzed showed that the team comprehends humanized care
\end{abstract}

\footnotetext{
${ }^{1}$ Graduanda em Enfermagem na Faculdade Fátima, Caxias do Sul, Rio Grande do Sul, Brasil. E-mail: sabrinavasquesag@yahoo.com.br

${ }^{2}$ Doutorado pelo Programa de Pós-graduação em Medicina e Ciências da Saúde da Pontifícia Universidade Católica do Rio Grande do Sul (PUCRS), Porto Alegre, Rio Grande do Sul, Brasil. Docente na Faculdade Fátima, Caxias do Sul, Rio Grande do Sul, Brasil. Docente na Faculdade da Serra Gaúcha (FSG), Caxias do Sul, Rio Grande do Sul, Brasil.
} 
and desire to acquire more knowledge. However, they are dissatisfied with work overload caused by overcrowding. Conclusion: the research evidenced that professionals face difficulties during their working hours due to the high service demand. Hence, the need to manage intense flow situations and the lack of professionals for the urgent and emergency sector is evident.

Keywords: Reception. Ethics in nursing. Patients. Nursing professionals.

\section{Introdução}

Humanizar a saúde consiste em reconhecer as práticas do convívio entre o profissional e o cliente, apoiando-se no respeito e confiança, possibilitando, assim, um acolhimento mais justo e mais digno para o paciente. (1) Desta forma, a Política Nacional de Humanização (PNH), inserida em 2003 pelo Ministério da Saúde (MS), descreve que a humanização se constitui no reconhecimento dos diversos sujeitos envolvidos no processo de produção de saúde: usuários, trabalhadores e gestores. ${ }^{(2)}$

Em complemento à PNH, no ano de 2004, o Ministério da Saúde produziu a cartilha sobre a Política Nacional de Humanização da Atenção e Gestão do Sistema Único de Saúde (PNHA GSUS), que regulamenta o acolhimento com avaliação e classificação de risco como plano de ação do trabalho na atenção e construção da saúde, em especial nos serviços de urgência. ${ }^{(3)}$

Esse atendimento consiste no acolhimento do usuário, desde sua entrada, responsabilizandose totalmente por ele, escutando suas queixas, aflições e angústias, assegurando resolutividade e a comunicação com os outros serviços de saúde para o seguimento da assistência, quando necessário. ${ }^{(4)}$

Porém, os serviços de emergência têm apresentado outra realidade: uma delas é o aumento da capacidade do serviço, que tem como resultado a sobrecarga de atividades para os profissionais que trabalham nessas unidades, além de levar a exaustão tanto para o trabalhador do setor quanto para o paciente. ${ }^{(5)}$

Consequentemente, essa busca ocasiona uma enorme tensão por parte tanto de usuários em reivindicar um bom atendimento, como de funcionários ao esforçar-se para dar conta do número dos atendimentos e ocorrências que aparecem a cada instante na unidade. Com isso, o cuidado se torna defasado e a qualidade do atendimento diminui à medida que aumenta a busca no setor de urgência e emergência. ${ }^{(6)}$

Além disso, conforme um estudo realizado em um Pronto-socorro Adulto (PSA) do Hospital de Clínicas da Universidade Federal do Triângulo Mineiro (HC-UFTM), os profissionais reclamam devido à falta de capacitação contínua para atuarem no setor de urgência e emergência e destacam a necessidade de treinamentos com os funcionários antes de sua inserção nos serviços de emergência, para que, assim, comecem aptos e adaptados à rotina da unidade. ${ }^{(7)}$

Todavia, de acordo com Lima Neto, ${ }^{(8)}$ ainda há muitos impedimentos para serem enfrentados, aprendizados contínuos e desenvolvimento dos novos sistemas. Com isso, torna-se necessário o envolvimento de administradores, trabalhadores e pacientes, uma vez que somente com a união de todos a consolidação e evoluções dessas diretrizes serão atingidas.

A partir do exposto, a equipe de enfermagem necessita de acesso às informações que permeiam o processo do atendimento humanizado, além de suporte necessário para realizar suas funções e treinamento para situações de fluxo intenso, visto que a demanda de atendimento nas portas emergenciais tem aumentado cada vez mais, o que tem ocasionado a superlotação do serviço e, por consequência, a queda na qualidade da assistência prestada.

\section{Material e Método}

Trata-se de um estudo descritivo, com abordagem quantitativa, que buscou conhecer a visão da equipe de enfermagem sobre o atendimento humanizado no setor de urgência e 
emergência, bem como as dificuldades encontradas no processo de trabalho; a mesma foi realizada em uma instituição privada localizada no Sul do Brasil.

Os critérios de inclusão foram possuir idade superior a 18 anos, mais de três meses de empresa e aceitar participar voluntariamente do estudo. E os critérios de exclusão foram não responder todas as perguntas do questionário e estar em afastamento, férias ou licença maternidade.

A coleta dos dados foi realizada por meio de um questionário contendo 20 perguntas, o qual foi elaborado pelos autores e aplicado no mês de março de 2020. Os profissionais que participaram da pesquisa foram abordados individualmente nos seus respectivos turnos de trabalho, e somente após aceitarem participar do estudo foi entregue o Termo de Consentimento Livre e Esclarecido (TCLE), em duas vias, e, posteriormente, o questionário. Considerando os critérios de inclusão e exclusão, a amostra foi composta por 16 questionários válidos.

Após o recolhimento do material, os dados foram analisados e organizados em tabelas, os quais foram computados no programa SPSS ${ }^{\circledR}$ - Statistical Package for the Social Sciences (SPSS $\left.{ }^{\circledR} 20\right)$. Na pergunta número 17 de cada questionário estava especificada a opção “outros”, onde os participantes poderiam mencionar suas opiniões. Desta forma, nos resultados utilizou-se a classificação dos questionários Q01 e Q02, consequentemente a fim de detalhar a questão e classificar os questionários por participantes.

A pesquisa ocorreu conforme os aspectos éticos, respeitando a Resolução da Diretoria Colegiada $n^{\circ}$ 466/12 de 2012 do Conselho Nacional de Saúde (CNS), tendo início somente após aprovação do Comitê de Ética em Pesquisa (CEP) e mediante Certificado de Apresentação para Apreciação Ética (CAAE) número 27109019.0.0000.5523.

\section{Resultados}

Dos profissionais, ao todo 17 participaram da pesquisa, no entanto, um questionário foi excluído devido ao fato de não terem sido respondidas todas as perguntas. Destarte, foram incluídos 16 questionários no estudo. Em relação ao sexo, 14 $(87,50 \%)$ participantes eram mulheres e $2(12,50 \%)$ eram homens. Referente à idade, 7 (43,75\%) dos participantes possuíam a média de \pm 18 a 30 anos. Relativo à escolaridade dos sujeitos envolvidos, $10(62,50 \%)$ eram técnicos de enfermagem, 3 $(18,75 \%)$ enfermeiros, e somente $3(18,75 \%)$ dos enfermeiros possuíam pós-graduação. A média do tempo de empresa dos participantes foi de \pm 1 ano, dos quais 7 (43,75\%) tinham 1 ano; 3 (18,75\%), 1 a 3 anos; 3 (18, 75\%), 3 a 10 anos; e 3 (18,75\%), 11 anos ou mais; e 16 (100\%) participantes possuíam carga horária de 220 horas/mês (Tabela 1).

Tabela 1 - Perfil profissional da equipe de enfermagem do setor de urgência e emergência de uma instituição hospitalar de Caxias do Sul - 2020.

\begin{tabular}{lcc}
\hline Perfil dos respondentes & n & \% \\
\hline 1. Gênero & 14 & 87,50 \\
Feminino & 2 & 12,50 \\
Masculino & & \\
2. Faixa etária & 7 & 43,75 \\
De 18 a 30 anos & 5 & 31,25 \\
De 31 a 40 anos & 2 & 12,50 \\
De 41 a 50 anos & 2 & 12,50 \\
51 anos ou mais & & \\
3. Escolaridade & 10 & 62,50 \\
Técnico de enfermagem & 3 & 18,75 \\
Enfermeiro & 3 & 18,75 \\
Pós-graduado & & \\
4. Tempo de instituição & 7 & 43,75 \\
Até 1 ano & 3 & 18,75 \\
De 1 a 3 anos & 3 & 18,75 \\
De 3 a 10 anos & 3 & 18,75 \\
11 anos ou mais & & \\
5. Carga horária trabalhada & 16 & 100,00 \\
220 horas mensais & & \\
\hline
\end{tabular}

Fonte: Dados da pesquisa

Conforme os dados apresentados na Tabela 2, foi possível observar que os funcionários reconhecem o significado do atendimento humanizado, 
pois dos 16 entrevistados, $13(81,25 \%)$ entendem que o paciente é um ser humano, 16 (100\%) participantes percebem que o mesmo deve ser acolhido desde a entrada até a saída do ambiente hospitalar, 9 participantes $(56,25 \%)$ reconhecem que o atendimento humanizado ameniza a dor e o sofrimento e 16 participantes (100\%) acreditam que a humanização no atendimento contribui para a melhora do paciente. Porém, conforme a questão 10 (dez), 9 (56,25\%) dos participantes evidenciam uma insatisfação em relação à alta demanda de atendimentos e impaciência por parte do paciente, e $2(12,50 \%)$ mencionam a falta de qualidade no atendimento (Tabela 2).

Tabela 2 - Avaliação pela equipe de enfermagem dos aspectos relacionados ao atendimento humanizado no setor de emergência de uma instituição hospitalar de Caxias do Sul - 2020.

\begin{tabular}{|c|c|c|}
\hline Perguntas & n & $\%$ \\
\hline \multicolumn{3}{|l|}{ 6. O que você entende por humanização da assistência de enfermagem? } \\
\hline É prestar um bom atendimento realizando boas técnicas. & 9 & 56,25 \\
\hline $\begin{array}{l}\text { É a valorização dos diferentes sujeitos implicando no processo de produção de saúde: } \\
\text { usuários, trabalhadores e gestores. }\end{array}$ & 6 & 37,50 \\
\hline É entender que o paciente/cliente é um ser humano. & 13 & 81,25 \\
\hline \multicolumn{3}{|l|}{ 7. Quando uma pessoa procura por atendimento você deve: } \\
\hline Prestar um atendimento acolhedor desde a entrada até a saída. & 16 & 100,0 \\
\hline Realizar atendimento o mais rápido possível para não deixá-la impaciente. & 4 & 25,00 \\
\hline Ouvir sua queixa. & 5 & 31,25 \\
\hline Tentar amenizar seu sofrimento. & 7 & 43,75 \\
\hline Solicitar que aguarde seu atendimento, pois o setor normalmente é lotado. & 2 & 12,50 \\
\hline \multicolumn{3}{|l|}{$\begin{array}{l}\text { 8. Você acredita que o atendimento humanizado pode influenciar na melhora } \\
\text { do quadro clínico de um paciente? }\end{array}$} \\
\hline Sim. & 16 & 100,0 \\
\hline \multicolumn{3}{|l|}{ 9. Na sua opinião, qual o motivo para se realizar um atendimento humanizado? } \\
\hline Troca e construção de saberes. & 4 & 25,00 \\
\hline Benefícios para todos os envolvidos, inclusive colaboradores. & 7 & 43,75 \\
\hline Contribui para a eficácia do cuidado ao paciente. & 11 & 68,75 \\
\hline Forte relação com a ética. & 4 & 25,00 \\
\hline Facilidade em ganhar a confiança do paciente. & 3 & 18,75 \\
\hline \multicolumn{3}{|l|}{$\begin{array}{l}\text { 10. Qual motivo está mais relacionado com a insatisfação do atendimento } \\
\text { no setor de urgência e emergência? }\end{array}$} \\
\hline Atraso no atendimento. & 6 & 37,50 \\
\hline Impaciência por parte do paciente. & 9 & 56,25 \\
\hline Falta de qualidade no atendimento. & 2 & 12,50 \\
\hline Setor com alta demanda. & 9 & 56,25 \\
\hline \multicolumn{3}{|l|}{$\begin{array}{l}\text { 11. Por que o atendimento humanizado deve ser priorizado no setor de urgência } \\
\text { e emergência? }\end{array}$} \\
\hline Porque ameniza a dor e o sofrimento. & 9 & 56,25 \\
\hline Por ser um setor onde o paciente está fragilizado e com medo. & 9 & 56,25 \\
\hline Porque é um setor com grande índice de estresse e demanda de trabalho. & 3 & 18,75 \\
\hline É bom para todos os envolvidos e melhora o ambiente de trabalho. & 5 & 31,25 \\
\hline Deve ser priorizada a humanização porque o paciente deve ser respeitado. & 3 & 18,75 \\
\hline
\end{tabular}

Fonte: Dados da pesquisa 
As informações apresentadas na Tabela 3 evidenciam que $13(81,25 \%)$ dos profissionais já receberam informação ou treinamentos referentes ao atendimento humanizado. Todavia, 7 (43,75\%) dos participantes informaram que os treinamentos foram externos. Treze (13) $(81,25 \%)$ expressaram desejo de adquirir mais conhecimentos em relação à humanização no atendimento e $16(100 \%)$ consideraram importante obter mais conhecimento, em razão de acreditarem que a enfermagem pode se equivocar na classificação de riscos. Além disso, $16(100 \%)$ participantes responderam que a equipe demonstra interesse em resolver os problemas dos pacientes e 14(87,50\%) consideram que mesmo com agitação do setor pode-se realizar um atendimento humanizado. No entanto, na questão 17 evidenciouse que $6(37,50 \%)$ desses profissionais encontram dificuldades com a falta de recursos no setor de trabalho e $2(12,50 \%)$ mencionam dificuldades de relacionamento com usuário (Tabela 3 ).

Tabela 3 - Avaliação do conhecimento e a visão dos profissionais em relação à humanização no atendimento no setor de urgência e emergência. Caxias do Sul - 2020.

\begin{tabular}{|c|c|c|}
\hline Perguntas & $\mathbf{n}$ & $\%$ \\
\hline \multicolumn{3}{|c|}{$\begin{array}{l}\text { 12. Já recebeu algum tipo de informação ou treinamento sobre o atendimento } \\
\text { humanizado? }\end{array}$} \\
\hline Sim. & 13 & 81,25 \\
\hline Não. & 3 & 18,75 \\
\hline \multicolumn{3}{|l|}{ 12a. Se você marcou SIM, qual a fonte de informação? } \\
\hline Televisão, rádio, jornal e internet. & 4 & 25,00 \\
\hline Treinamentos externos. & 7 & 43,75 \\
\hline Treinamentos no hospital. & 6 & 37,50 \\
\hline Campanhas educativas. & 4 & 25,00 \\
\hline Outros: livros. & 1 & 6,25 \\
\hline \multicolumn{3}{|c|}{$\begin{array}{l}\text { 13. Você teria o desejo de se aprofundar mais no assunto através de livros, artigos, } \\
\text { palestras e cursos? }\end{array}$} \\
\hline Sim. & 13 & 81,25 \\
\hline Sim, mas não tenho tempo. & 2 & 12,50 \\
\hline Não tenho interesse. & 1 & 6,25 \\
\hline \multicolumn{3}{|c|}{$\begin{array}{l}\text { 14. Você acha importante um profissional se atualizar e adquirir } \\
\text { novos conhecimentos? }\end{array}$} \\
\hline Sim. & 16 & 100,0 \\
\hline \multicolumn{3}{|c|}{$\begin{array}{l}\text { 15. Com relação ao atendimento com classificação de risco você acredita } \\
\text { que a enfermagem possa se equivocar na classificação? }\end{array}$} \\
\hline Sim. & 16 & 100,0 \\
\hline \multicolumn{3}{|c|}{$\begin{array}{l}\text { 16. No geral, a equipe de enfermagem demonstra interesse sincero em resolver } \\
\text { os problemas do paciente? }\end{array}$} \\
\hline Sim. & 16 & 100,0 \\
\hline \multicolumn{3}{|c|}{ 17. Qual a dificuldade que você encontra em aplicar o processo de humanização? } \\
\hline Falta de recursos no meu local de trabalho. & 6 & 37,50 \\
\hline Dificuldade de relacionamento com o paciente/cliente. & 2 & 12,50 \\
\hline Carga horária excessiva. & 2 & 12,50 \\
\hline Não sei o que é. & 1 & 6,25 \\
\hline
\end{tabular}


Continuação

18. Você acredita que mesmo com a agitação e demanda de serviço no setor de urgência, pode-se realizar um atendimento humanizado?

Sim.

Não.

É muito difícil, mas não é impossível.

19. Qualifique o atendimento no setor de urgência e emergência em relação ao atendimento humanizado:

Excelente.

Bom.

Regular.

20. Na sua opinião, você consegue realizar um atendimento humanizado?

Sim.

$14 \quad 87,50$

Às vezes.

12,50

Fonte: Dados da pesquisa

Na sequência, podemos observar as opiniões mencionadas na pergunta 17 dos questionários, a qual solicitava a maior dificuldade do profissional em aplicar o processo de humanização:

Poucos funcionários para uma grande demanda de pacientes. (Q02).

Alta demanda. (Q05).

Setor com muita demanda de pacientes $e$ poucos profissionais para prestar assistência, dificultando dar devida atenção a todos os pacientes. (Q07).

Sensibilizar os colaboradores. (Q10).

Falta de humanização do empregador com os empregados. (Q11).

Quando há muita demanda, não temos tempo para dar atenção ao paciente. (Q14).

\section{Discussão}

Ficou evidenciado que a maioria dos profissionais era do sexo feminino, em concordância com Costa,${ }^{(9)}$ que a prática da enfermagem é manifestada como uma atividade reconhecida pela atuação das mulheres. A média de idade dos participantes $( \pm 18$ a 30 anos) nos leva ao entendimento que muitos dos jovens demonstram interesse pelas áreas de alta complexidade na saúde. Contudo, poucos realizam especialização após a graduação, pois somente metade dos enfermeiros era especializada. Atualmente, o enfermeiro, ao iniciar sua carreira, depara-se com um mercado de trabalho que demanda crescente inovação e competência profissional, no intuito de aperfeiçoar a qualidade do atendimento. ${ }^{(10)}$

Outro resultado identificado foi o tempo de empresa, cuja média era de ( \pm 1 ano). São muitos os obstáculos avistados ao começar no trabalho; todavia, cada funcionário demonstra uma forma diferenciada de ultrapassar seus desafios. Assim, obter experiência ao longo do tempo e buscar auxílio de colegas com mais conhecimento são atitudes significativas no sentido de vencer algumas dificuldades que serão encontradas. ${ }^{(11)}$

Por conseguinte, o estudo demonstrou que os profissionais compreendem o significado do atendimento humanizado e entendem que o paciente é um ser humano, devendo esse ser acolhido desde a entrada até a saída do ambiente hospitalar, uma vez que a humanização ameniza a dor e o sofrimento e contribui para a melhora do paciente. Em vista disso, obteve-se um bom conceito em relação à percepção da equipe frente ao atendimento humanizado, pois, segundo o Ministério da Saúde, o acolhimento é uma das metodologias utilizadas juntamente com o esquema de classificação de 
risco, os quais têm por finalidade a humanização do serviço e a melhoria do atendimento. ${ }^{(12)}$

No entanto, os participantes se queixaram da alta demanda no atendimento e mencionam o atraso devido à superlotação do serviço, além de relatarem a impaciência por parte do paciente em aguardar atendimento. Torna-se, portanto, necessária a organização do serviço, já que a humanização do cuidado à saúde demanda atenção a diversas questões, devendo essas serem voltadas e ordenadas por uma filosofia organizacional, cujas convicções devem estar especificadas e possíveis de serem executadas na prática. ${ }^{(13)}$

Logo, os enfermeiros mencionam que um dos maiores obstáculos enfrentados na aplicação do acolhimento humanizado e na assistência com qualidade é devido à elevada demanda de atendimentos $[\ldots] ;{ }^{(8)}$ e que essa demasiada busca ocasiona uma grande apreensão por parte tanto de usuários em reivindicar um bom atendimento, como de funcionários ao esforçar-se para dar conta do número dos atendimentos e ocorrências que aparecem a cada instante na unidade $[. . .]^{(6)}$

Desse modo, é possível evidenciar a cascata de conflitos que a alta demanda ocasiona no setor, uma vez que, mesmo com todo o conhecimento dos profissionais e esforço do Ministério da Saúde em implantar a classificação de risco como forma de desobstruir as portas de emergência, ainda assim os serviços de emergência têm apresentado outra realidade, onde uma delas é o aumento da capacidade do serviço, que tem como resultado a sobrecarga de atividades para os profissionais que trabalham nessas unidades, além de levar a exaustão tanto para o trabalhador do setor quanto para o paciente. ${ }^{(5)}$

Confirma-se, dessa forma, que os protocolos de classificação de risco são ferramentas que estruturam a avaliação, sendo um mecanismo útil e indispensável, porém não o bastante, pois o mesmo não tem intenção de analisar os aspectos subjetivos, afetivos, sociais e culturais, cujo entendimento é essencial para uma atenciosa análise de risco e da fragilidade de cada usuário que busca o atendimento de urgência. Ou seja, o protocolo não substitui o convívio, a comunicação, o ouvir, o respeito, isto é, o acolhimento do paciente e de sua queixa, a fim de avaliar seu estado. ${ }^{(14)}$

Consequentemente, ainda há muitos impedimentos para serem enfrentados, aprendizados contínuos e desenvolvimento dos novos sistemas, fazendo-se necessário o envolvimento de administradores, trabalhadores e pacientes, porque somente com a união de todos a consolidação e evoluções dessas diretrizes serão atingidas, ${ }^{(8)}$ dado que as dificuldades para a execução desse acolhimento estão associadas ao sentimento de impotência, relacionadas às mudanças do estado clínico do usuário que aguarda por acolhimento e ao esgotamento decorrente das ações causadas pelos mesmos ao não aceitarem a avaliação realizada pelo enfermeiro. ${ }^{(15)}$

Diante dos fatos, a assistência realizada aos pacientes no Serviço Hospitalar de Emergência (SHE) torna-se uma condição cada vez mais estudada no mundo; ligado a isso, a responsabilidade da enfermagem nesse setor tem crescido diariamente por ser esta uma função muito importante nos últimos anos, devido às diversas transformações socioepidemiológicas que vêm acontecendo com o passar do tempo. ${ }^{(16-17)}$

Desta maneira, compreende-se a extrema importância do desenvolvimento de ações nos serviços de urgência e emergência que proporcionem a humanização, não apenas para os pacientes, mas também para os profissionais de saúde, ${ }^{(18)}$ porque a sobrecarga de trabalho, o salário baixo e a falta de valorização como aspectos relacionados às falhas no serviço prejudicam a qualidade do cuidado prestado aos usuários atendidos no setor de urgência e emergência e essas condições podem provocar aos profissionais de enfermagem desinteresse e incertezas e desenvolver uma queda no desempenho do profissional. ${ }^{(19)}$

\section{Conclusão}

Por meio do presente estudo, tornou-se possível conhecer a visão da enfermagem sobre o atendimento humanizado na unidade de urgência 
e emergência, uma vez que o mesmo deixou claro que, além da equipe ter conhecimento sobre o atendimento humanizado e expressar desejo em adquirir mais conhecimento sobre o assunto, reconhece que a humanização faz parte do cuidado e da assistência de enfermagem.

Entretanto, a pesquisa também ressaltou as dificuldades que os profissionais enfrentam durante sua jornada de trabalho, evidenciando a insatisfação dos mesmos em relação à alta demanda de atendimento no setor de urgência e emergência, a falta de funcionários para realizarem os atendimentos e o despreparo de alguns empregadores em lidar com o empregado no serviço de emergência. Sugerese, assim, a revisão do número de profissionais, a implementação de ações voltadas para o fluxo intenso e treinamentos com intuito de reforçar a ética no ambiente de trabalho.

Desse modo, para se realizar um atendimento de qualidade em um setor de alta complexidade como o setor de emergência, é notório que, além da disposição da equipe em realizar um atendimento humanizado, também é necessária a reestruturação do serviço para que os mesmos sejam capazes de desenvolver suas atribuições.

\section{Referências}

1 Rocha TRA, Pinto FO. A humanização na assistência de enfermagem em unidades de urgência e emergência. Rev Científ Interdisciplinar. 2016;3(3):46-62. doi: 10.17115/23588411/v3n3a4.

2 Ministério da Saúde. Núcleo Técnico da Política Nacional de Humanização. HumanizaSUS Acolhimento com Avaliação e classificação de risco: um paradigma ético-estético no fazer em saúde. Brasília: Ministério da Saúde; 2004.

3 Oliveira GN, Vancini-Campanharo CR, Okuno MFP, Batista REA. Nursing care based on risk assessment and classification: agreement between nurses and the institutional protocol. Rev Latino-Am Enfermagem [Internet]. 2013 [citado 2020. jan 20];21(2). Disponível em: http://www.scielo.br/pdf/rlae/v21n2/pt_01041169-rlae-21-02-0500.pdf
4 Gibaut MDAM, Hori LMR, Freitas KS, Mussi FC. Comfort of the patient's family in an Intensive Care Unit related to welcoming. Rev Esc Enferm. USP. 2013;47(5):1114-21.

5 Simões CG, Urbanetto JS, Figueiredo AEPL. Ação interdisciplinar em serviços de urgência e emergência: uma revisão integrativa. Rev Ciênc Saúde. 2013;6(2):127-34.

6 Nascimento ERP, Hilsendeger BR, Neth C, Belaver GM, Bertoncello KCG. Classificação de risco na emergência: Avaliação da equipe de enfermagem. Rev Enferm UERJ. 2011;19(1): 84-8.

7 Rocha NHG, Lemos RCA. Atitudes da equipe e qualidade da assistência de enfermagem em um pronto socorro adulto. Rev Enferm Atenção Saúde. 2017;6(2):106-17. doi: 10.18554/reas. v6i2.1842.

8 Lima AV Neto, Nunes VMA, Fernandes RL, Barbosa IML, Carvalho GRP. Humanização e acolhimento em emergência hospitalar: fatores condicionantes sob o olhar dos enfermeiros. Rev Pesq Cuid Fundam. 2013;5(4):519-28.

9 Costa JRA, Lima JV, Almeida PC. Stress no trabalho do enfermeiro. Rev Esc Enferm USP. 2003;37(3):63-71. doi: 10.1590/S008062342003000300008 .

10 Manarin AP, Bortoleto CB, Ferreira-Sae MCS. Perspectivas do egresso de enfermagem frente ao mercado de trabalho. Ens Ciênc. 2009;13(1):93-105.

11 Souza FA; Paiano M. Desafios e dificuldades enfrentadas pelos profissionais de Enfermagem em início de carreira. REME Rev Min Enferm [Internet]. 2011 [citado 2020 jan 15];15(2):26773. Disponível em: http://www.reme.org.br/ artigo/detalhes/35

12 Ministério da Saúde. Secretaria de Atenção à Saúde. Departamento de Ações Programáticas e Estratégicas. Política Nacional de Normalização. Atenção hospitalar. Brasília: Ministério da Saúde; 2013.

13 Andrade LM, Martins EC, Caetano JA, Soares E, Beserra EP. Atendimento humanizado nos serviços de emergência hospitalar na percepção 
do acompanhante Rev Eletron Enferm [Internet]. 2009 [citado 2020 jun 2019];11(1): 151-7. Disponível em: http://www.fen.ufg.br/revista/ v11/n1/v11n1a19.htm

14 Ministério da Saúde (MS). Política Nacional de Humanização da Atenção e Gestão do SUS. Acolhimento e classificação de risco nos serviços de urgência [Internet]. Brasília: Ministério da Saúde; 2009. [citado 2020 jan 20]. Disponível em: http://bvsms.saude.gov. br/bvs/publicacoes/acolhimento_classificaao_ risco_servico_urgencia.pdf

15 Acosta AM, Duro CLM, Lima MADS. Atividades do enfermeiro nos sistemas de triagem/classificação de risco nos serviços de urgência: revisão integrativa. Rev Gaúcha Enferm. 2012;33(4):181-90.

16 Santos JLG, Lima MADS, Pestana AL, Garlet ER, Erdmann AL. Desafios para a gerência do cuidado em emergência na perspectiva de enfermeiros. Acta Paul Enferm. 2013;26(2):136-43. doi: 10.1590/S0103-2100 2013000200006.

17 Avelino FVSD, Leite ARF, Fernandes MA, Avelino FPD, Madeira MZA, Souza LEN. Estresse em enfermeiros do setor de urgência e emergência. Rev Enferm UFPI [Internet]. 2013 [citado 2020 jan 15];2(3). Disponível em: http://www.scielo.br/scielo.php?pid=S0103

18 Sousa PCC, Silva OAD, Ferreira PQC, Sousa DJ, Lago EC, Sousa MDCP. Humanização da assistência de enfermagem em unidade de urgência e emergência. Rev Interdisciplinar [Internet]. 2015 [citado 2020 jun 15];8(1):204-10. Disponível em: http:// revistainterdisciplinar.uninovafapi.edu.br/ index.php/revinter/article/view/576

19 Furtado BMASM, Araújo Júnior JLC. Percepção de enfermeiros sobre condições de trabalho em setor de emergência de um hospital. Acta Paul Enferm. 2010;23(2):169-74. 
Cézar, S. V.; Souza, J. S. M. 